\title{
High-fidelity simulation for extracorporeal membrane oxygenation training, utile or futile?
}

\author{
Carlo Banfi ${ }^{1,2,3}$, Karim Bendjelid ${ }^{2,3,4}$, Raphaël Giraud ${ }^{2,3,4}$ \\ ${ }^{1}$ Division of Cardiovascular Surgery, Geneva University Hospitals, Geneva, Switzerland; ${ }^{2}$ Faculty of Medicine, University of Geneva, Switzerland; \\ ${ }^{3}$ Geneva Hemodynamic Research Group, Geneva, Switzerland; Intensive Care Service, Geneva University Hospitals, Geneva, Switzerland \\ Correspondence to: Raphaël Giraud, MD, PhD. Intensive Care Service, Geneva University Hospitals 4, Rue Gabrielle Perret-Gentil, CH-1211 Geneva \\ 14, Switzerland. Email: Raphael.Giraud@hcuge.ch. \\ Provenance: This is an invited Editorial commissioned by Section Editor Dr. Lei Huang (Cardiac Center of Tianjin Third-Central Hospital, Tianjin, China). \\ Comment on: Zakhary BM, Kam LM, Kaufman BS, et al. The utility of high-fidelity simulation for training critical care fellows in the management of \\ extracorporeal membrane oxygenation emergencies: a randomized controlled trial. Crit Care Med 2017;45:1367-73.
}

Submitted Oct 03, 2017. Accepted for publication Oct 11, 2017.

doi: $10.21037 /$ jtd.2017.10.54

View this article at: http://dx.doi.org/10.21037/jtd.2017.10.54

Extracorporeal membrane oxygenation (ECMO) allows a new approach for the intensive care management of acute cardiac and/or respiratory failure in patients who are not responsive to conventional treatment $(1,2)$. While ECMO volume and number of ECMO centers increased more than 10 -fold over the past decade, the management of these patients remains highly challenging as ECMO is one of the trickiest therapies available in the ICU. Indeed, the present technique requires a comprehensive knowledge of cardiopulmonary physiology, expertise with complex circuit components, and skills to rapidly respond to potential life-threatening complications. From cannulation to management of ongoing ECMO and finally weaning from this support, each step should be mastered by the doctors involved in the management of these patients. In this regard physicians' experience is crucial as expertise in this technology requires extensive and continuous training (3).

ECMO organizations recommend practitioner education via lectures with water-drills or animal testing. However, while water-drills offer limited opportunity for real-time troubleshooting, and the use of big animals is difficult, expensive (4) and not always equivalent to human pathophysiology. Moreover, despite the increasing requirement for ECMO specialists and the needs on existing facilities, training offers in ECMO are limited. Thus simulation-based training represents a valuable tool in intensive care education (5), offering the opportunity to practice technical skills repeatedly and to become proficient in high-risk, low-frequency events while avoiding harm to patients (6).

In a recent article published in Critical Care Medicine (7), the authors compared ECMO training based on traditional water-drill with simulation-based training in a uniform randomized controlled trial. Elegantly, the authors demonstrated that simulation-based ECMO training is effective and superior to traditional water-drill-based training with reductions in times to critical actions for emergency circuit management. And, interestingly, these benefits were maintained over one-year period. Three scenarios were developed to reflect complications an ECMO practitioner is expected to experiment: "scenario 1: recirculation (Sim1), scenario 2: pump failure ( $\operatorname{Sim} 2$ ) and scenario 3: access insufficiency (Sim3)". Twenty-one pulmonary/critical care fellows had a pre-intervention simulated ECMO emergency (Sim1). Then they were randomized into two groups: 10 in the simulation group and 11 in the traditional group. Three teaching scenarios were performed by each group, whether via highfidelity simulation or water-drills. Participants returned after 6 weeks and after 1 year for two simulated ECMO emergencies (Sim2 and $\operatorname{Sim} 3$ ). As $\operatorname{Sim} 2$ was a situation experienced during training, $\operatorname{Sim} 3$ was specifically novel. For resolution of each scenario, a critical action was necessary and pre-identified for timing. Authors defined as the 
primary outcome, the time needed to respond and achieve these critical actions. Results showed that the 2 groups had similar scenario scores and times to critical action on Sim1. At 6 weeks, both groups had similar scenario scores on Sim2, but the simulation group scored significantly higher on Sim3. Times to performed critical actions were significantly shorter in the simulation group during Sim2 and $\operatorname{Sim} 3$ and all findings persisted for 1 year.

The present study is the first randomized controlled trial evaluating the utility of simulation in ECMO education with objective, measurable, clinically relevant and longterm outcomes. It provided insights into the useful clinical application of simulation training as a valuable tool to dispense ECMO training. First, it allows the safe development of relevant clinical skills without direct impact on patients where errors may have direct consequences on mortality and morbidity. Second, it permits the practice or either unusual or infrequent life-threatening events (8). Finally, it also allows the assessment of ECMO experience prior to clinical engagement and also meets the continuing educational requirements of an ECMO practitioner.

In addition to allowing the increase of technical skills, simulation-based training has also been shown to provide essential cognitive and behavioral skills when compared to theoretic teaching (9). Moreover, it improves team working quality and safety (10). Benefits of using simulation training for ECMO have been demonstrated in a number of previous studies. In cases of ECMO emergencies, Anderson et al. confirmed a decreased amount of errors after simulationbased training (9). An improvement in confidence in ECMO practitioners receiving simulation training has also been confirmed by Burkhart et al. (8).

In the past ten years, simulation techniques have been improved from low fidelity to high fidelity models that now are able to mimic human responses in a very realistic way. Evidence supports a timely detection of emergency circuit management and long-term maintenance of skills learned by simulation training. Adding simulation to fellowship programs and postgraduate formation seems to be a feasible and reliable ECMO postgraduate learning. It will certainly improve and speed up the theoretical and the practical education that increase physician expertise (11). However, evidence is lacking whether the use of simulators is associated with better patient outcome and research is needed in this field to define the standards of high fidelity simulation in aspects of ECMO intensive care training.

\section{Acknowledgements}

None.

\section{Footnote}

Conflicts of Interest: The authors have no conflicts of interest to declare.

\section{References}

1. Abrams D, Brodie D. Extracorporeal membrane oxygenation for adult respiratory failure: 2017 update. Chest 2017;152:639-49.

2. Ouweneel DM, Schotborgh JV, Limpens J, et al. Extracorporeal life support during cardiac arrest and cardiogenic shock: a systematic review and meta-analysis. Intensive Care Med 2016;42:1922-34.

3. Brum R, Rajani R, Gelandt E, et al. Simulation training for extracorporeal membrane oxygenation. Ann Card Anaesth 2015;18:185-90.

4. Brazzi L, Lissoni A, Panigada M, et al. Simulation-based training of extracorporeal membrane oxygenation during H1N1 influenza pandemic: the Italian experience. Simul Healthe 2012;7:32-4.

5. Sekiguchi H, Tokita JE, Minami T, et al. A prerotational, simulation-based workshop improves the safety of central venous catheter insertion: results of a successful internal medicine house staff training program. Chest 2011;140:652-8.

6. Akaike M, Fukutomi M, Nagamune M, et al. Simulationbased medical education in clinical skills laboratory. J Med Invest 2012;59:28-35.

7. Zakhary BM, Kam LM, Kaufman BS, et al. The utility of high-fidelity simulation for training critical care fellows in the management of extracorporeal membrane oxygenation emergencies: a randomized controlled trial. Crit Care Med 2017;45:1367-73.

8. Burkhart HM, Riley JB, Lynch JJ, et al. Simulation-based postcardiotomy extracorporeal membrane oxygenation crisis training for thoracic surgery residents. Ann Thorac Surg 2013;95:901-6.

9. Anderson JM, Murphy AA, Boyle KB, et al. Simulating extracorporeal membrane oxygenation emergencies to improve human performance. Part II: assessment of technical and behavioral skills. Simul Healthc 2006;1:228-32. 
10. Burton KS, Pendergrass TL, Byczkowski TL, et al. Impact of simulation-based extracorporeal membrane oxygenation training in the simulation laboratory and clinical environment. Simul Healthc 2011;6:284-91.

Cite this article as: Banfi C, Bendjelid K, Giraud R. Highfidelity simulation for extracorporeal membrane oxygenation training, utile or futile? J Thorac Dis 2017;9(11):4283-4285. doi: $10.21037 /$ jtd.2017.10.54
11. Clau-Terre F, Sharma V, Cholley B, et al. Can simulation help to answer the demand for echocardiography education? Anesthesiology 2014;120:32-41. 